\title{
Postural tachycardia syndrome and suicide: what we don't know
}

\author{
Vidya Raj $^{1,2} \cdot$ Satish R. Raj ${ }^{3,4}$
}

Received: 6 February 2017/Accepted: 7 February 2017/Published online: 18 February 2017

(c) Springer-Verlag Berlin Heidelberg 2017

\begin{abstract}
"There are known knowns; there are things we know we know. We also know there are known unknowns; that is to say we know there are some things we do not know. But there are also unknown unknownsthe ones we don't know we don't know". -US Secretary of Defense Donald H. Rumsfeld (February 12,2002$)[1]$.
\end{abstract}

Suicide is notoriously difficult to study and even more difficult to predict, yet it imposes a major burden on society. The World Health Organization reports that suicide accounted for $1.4 \%$ of deaths worldwide in 2012 [2], but the prevalence of suicidal ideation (thinking about suicide) is much more common, at 9.2\% [3]. Suicide research efforts are plagued by difficulties in distinguishing which of the many people who think about suicide will successfully complete their suicide. This is because it has proven challenging to find predictors of repeated suicide attempts, and harder yet to find predictors of completed suicide [4].

Satish R. Raj

Satish.raj@ucalgary.ca

1 Department of Psychiatry, University of Calgary, Calgary, Canada

2 Department of Family Medicine, University of Calgary, Calgary, Canada

3 Department of Cardiac Sciences, Libin Cardiovascular Institute of Alberta, University of Calgary, GAC70 HRIC Building, 3280 Hospital Drive NW, Calgary, AB T2N 4Z6, Canada

4 Division of Clinical Pharmacology, Department of Medicine, Autonomic Dysfunction Center, Vanderbilt University Medical Center, Nashville, TN, USA
In this issue of Clinical Autonomic Research, Pederson and Brook have published the first paper examining the relationship between postural tachycardia syndrome (POTS) and suicide [5]. We applaud them for starting to systematically study this important subject, despite the complexity involved in understanding and interpreting this field. The authors used a four-item self-report questionnaire, the Suicidal Behaviors Questionnaire-Revised (SBQ-R) [6], which was developed to measure past suicidal behavior, and thoughts of suicide in particular. An SBQ-R score of $>7$ places an individual in a "high-risk" group which predicted correlation with a group of adult and adolescent psychiatric inpatients experiencing suicidal ideation or attempts. The authors found that a higher percentage of POTS patients had high suicide risk compared to control subjects (48 vs. 18\%). A greater proportion of POTS patients had threatened suicide (37 vs. $19 \%$ ), planned a suicide attempt (24 vs. $14 \%$ ), or attempted suicide (16 vs. $3 \%$ ) than control subjects. It is very important to note that the SBQ-R score is largely driven by suicidal ideation and is not predictive of future suicide attempts [6]. Based on these data alone, we cannot conclude that patients with POTS are more likely to actually complete suicide.

The meaning of a suicide attempt is also important. Did the attempter mean to die? Did they not care whether they died? Did they injure themselves? Or did they perhaps obtain a secondary gain, such as attention from a loved one? These are key issues, since not all attempts at selfinjury represent a true suicide attempt, yet patients may still classify them as suicide attempts when questioned. A classic example of this occurs in patients with borderline personality disorder who injure themselves under emotional distress. The only case where one can be sure that the attempter meant to die is a completed suicide.

Recent data suggest that suicidal ideation and attempts are different constructs [3]. Often-cited classic risk factors 
for suicide (including depression, hopelessness, psychiatric disorders, and impulsivity) are strongly associated with suicidal ideation but correlate weakly with suicide attempts.

What are the causal links that place patients with POTS at increased risk of suicidal ideation and possibly suicide attempts? The literature supports fixed risk factors for attempting suicide including female gender, young age (suicide is the second leading cause of death in those aged 15-29 years) or old age, and low educational attainment, as well as modifiable factors including psychiatric illness (particularly depression), substance use disorders, personality disorders, impulsivity, and unemployment [4, 7]. Medical illnesses such as visual impairment, neurological disease, and malignant disease have also been associated with increased odds of suicide in the elderly [8]. These data offer some insight into potential causal factors for the increase in suicidal ideation in POTS patients. Most POTS patients are young to middle-aged women with a chronic illness and reduced quality of life [9]. There may also be comorbid depression and anxiety, and personality disorders (that were not assessed in this study) that could affect susceptibility to suicidal ideation.

What does this mean for our POTS patients? Pederson and Brook's work clearly shows that POTS patients experience more significant mental distress, including suicidal ideation, compared with control subjects. It is also very concerning that significantly more POTS patients than control subjects report having attempted suicide. While we cannot conclude that patients with POTS are more likely to complete suicide than control subjects, this important question requires further investigation.

The data from Pederson and Brook [5] are concerning. While they have converted suicide risk in POTS from an "unknown unknown" to a "known unknown", we need to better understand whether POTS is associated with increased risk of future suicide attempts and completed suicide. We must also further investigate the factors causing increased suicidal ideation. By making this a "known known", we can target the right patients with treatment strategies that will reduce the risk of suicide in patients with POTS.

\section{Compliance with ethical standards}

Sources of funding This work was supported in part by the National Institutes of Health grants R01 HL102387 and UL1 TR000445, and by the Canadian Institutes of Health Research (CIHR; Ottawa, Canada) grant MOP142426.

\section{References}

1. Rumsfeld DH, Myers RB. DoD News Briefing-Secretary Rumsfeld and Gen. Myers. US Department of Defense; Last Updated: 2002 February 12; http://archive.defense.gov/Transcripts/Tran script.aspx?TranscriptID=2636. Accessed 1 Feb 2017

2. World Health Organization. Mental Health: Suicide. World Health Organization; Last Updated: 2017. http://www.who.int/mental_ health/prevention/suicide/suicideprevent/en/. Accessed 1 Feb 2017

3. Klonsky ED, May AM, Saffer BY (2016) Suicide, suicide attempts, and suicidal ideation. Annu Rev Clin Psychol 12:307-330

4. Beghi M, Rosenbaum JF, Cerri C, Cornaggia CM (2013) Risk factors for fatal and nonfatal repetition of suicide attempts: a literature review. Neuropsychiatr Dis Treat 9:1725-1736

5. Pederson C, Brook JB (2017) Health-related quality of life and suicide risk in postural tachycardia syndrome. Clin Auton Res. doi:10.1007/s10286-017-0399-5.

6. Osman A, Bagge CL, Gutierrez PM, Konick LC, Kopper BA, Barrios FX (2001) The Suicidal Behaviors Questionnaire-Revised (SBQ-R): validation with clinical and nonclinical samples. Assessment 8(4):443-454

7. Nock MK, Borges G, Bromet EJ et al (2008) Cross-national prevalence and risk factors for suicidal ideation, plans and attempts. Br J Psychiatry 192(2):98-105

8. Waern M, Rubenowitz E, Runeson B, Skoog I, Wilhelmson K, Allebeck P (2002) Burden of illness and suicide in elderly people: case-control study. BMJ 324(7350):1355

9. Bagai K, Song Y, Ling JF et al (2011) Sleep disturbances and diminished quality of life in postural tachycardia syndrome. J Clin Sleep Med 7(2):204-210 